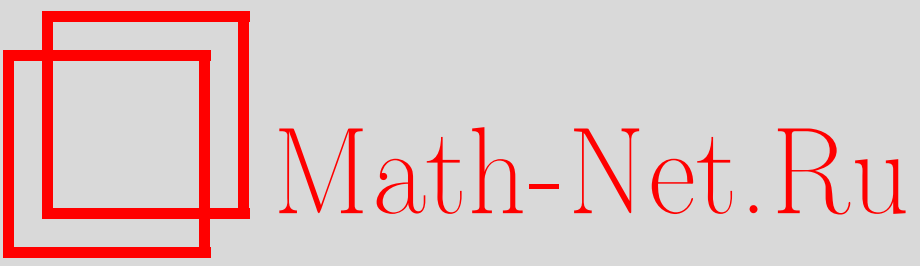

Р. А. Лелеков, Необходимые и достаточные условия сходимости условных математических ожиданий относительно сигма-алгебр, УМН, 1998, том 53, выпуск $6,255-256$

DOI: https://doi.org/10.4213/rm101

Использование Общероссийского математического портала Math-Net.Ru подразумевает, что вы прочитали и согласны с пользовательским соглашением

http://www. mathnet.ru/rus/agreement

Параметры загрузки:

IP: 3.80 .253 .173

26 апреля 2023 г., 15:42:02 


\title{
НЕОБХОДИМЫЕ И ДОСТАТОЧНЫЕ УСЛОВИЯ СХОДИМОСТИ УСЛОВНЫХ МАТЕМАТИЧЕСКИХ ОЖИДАНИЙ ОТНОСИТЕЛЬНО СИГМА-АЛГЕБР
}

\author{
Р. А. ЛЕЛЕКОВ
}

Tеорема. Пусть $(\Omega, \mathscr{F}, \mathrm{P})$ - вероятностное пространство $\xi_{n} \geqslant 0, n \geqslant 1,\left\{\xi_{n}\right\}_{n \geqslant 1}$ - последовательность случайных величин с $\mathrm{E}\left(\xi_{n} \mid \mathscr{G}\right)<\infty($ п.н. $), \xi_{n} \rightarrow \xi($ (п.н.) $и \mathscr{G} \subset \mathscr{F}$ - $\sigma$-подалгебра $\mathscr{F}$.

Тогда $\mathrm{E}\left(\xi_{n} \mid \mathscr{G}\right) \rightarrow \mathrm{E}(\xi \mid \mathscr{G})<\infty$ (п.н.) тогда и только тогда, когда для некоторой последовательности $\left\{c_{m}\right\}_{m \geqslant 1}, c_{m} \in \mathbb{R}^{+}, c_{m} \rightarrow \infty, c_{m+1}>c_{m}$,

$$
\sup _{n} \mathrm{E}\left(\xi_{n} I_{\left\{\xi_{n}>c_{m}\right\}} \mid \mathscr{G}\right) \underset{m \rightarrow \infty}{\longrightarrow} 0 \quad \text { (n.н.). }
$$

ЗАмЕчАнИЕ. В силу того, что последователшность $\sup \mathrm{E}\left(\xi_{n} I_{\left\{\xi_{n}>c_{m}\right\}} \mid \mathscr{G}\right)$ монотонно убывает п.н., последнее условие выполнено для произвольной монотонно стремящейся к бесконечности последовательности $\left\{c_{m}\right\}_{m \geqslant 1}$, если оно выполнено хотя бы для одной такой последовательности.

СлЕдСтвиЕ. Если $\mathscr{G}=(\varnothing, \Omega)$, mо $\mathrm{E}(\eta \mid \mathscr{G})=\mathrm{E} \eta($ п.н.) для произвольной случайной величинь $\eta$, и мы имеем следующий известный результат.

Если $0 \leqslant \xi_{n} \rightarrow \xi\left(\right.$ (п.н.) $c \mathrm{E} \xi_{n}<\infty$, mo

$$
\mathrm{E} \xi_{n} \rightarrow \mathrm{E} \xi<\infty \Longleftrightarrow \sup _{n} \mathrm{E}\left(\xi_{n} I_{\left\{\xi_{n}>c\right\}}\right) \underset{c \rightarrow \infty}{\longrightarrow} 0 .
$$

Последнее условие означает равномерную интегрируемость семейства случайньх величин $\left\{\xi_{n}\right\}_{n \geqslant 1}$.

ДоКАЗАТЕЛЬСТво тЕОРЕМЫ. Достаточность. Пусть задана некоторая последовательность $\{c m\}_{m \geqslant 1}$. Тогда (п.н.)

$$
\forall m \geqslant 1 \varlimsup \overline{\lim } \mathrm{E}\left(\xi_{n} \mid \mathscr{G}\right) \leqslant \sup _{n} \mathrm{E}\left(\xi_{n} I_{\left\{\xi_{n}>c_{m}\right\}} \mid \mathscr{G}\right)+\mathrm{E}(\xi \mid \mathscr{G})
$$

и $\varlimsup \mathrm{\operatorname {lim }} \mathrm{E}\left(\xi_{n} \mid \mathscr{G}\right) \leqslant \mathrm{E}(\xi \mid \mathscr{G}) \leqslant \underline{\lim } \mathrm{E}\left(\xi_{n} \mid \mathscr{G}\right)$ (п.н.), т.е. $\mathrm{E}\left(\xi_{n} \mid \mathscr{G}\right) \rightarrow \mathrm{E}(\xi \mid \mathscr{G})$ (п.н.).

Далее, (п.н.)

$$
\forall m \geqslant 1 \sup _{n} \mathrm{E}\left(\xi_{n} \mid \mathscr{G}\right) \leqslant \sup _{n} \mathrm{E}\left(\xi_{n} I_{\left\{\xi_{n} \leqslant c_{m}\right\}} \mid \mathscr{G}\right)+\sup _{n} \mathrm{E}\left(\xi_{n} I_{\left\{\xi_{n}>c_{m}\right\}} \mid \mathscr{G}\right) .
$$

Для произвольного фиксированного $\varepsilon$ выбираем $m \geqslant 1$ так, что

$$
\begin{gathered}
\sup _{n} \mathrm{E}\left(\xi_{n} I_{\left\{\xi_{n}>c_{m}\right\}} \mid \mathscr{G}\right)<\varepsilon, \\
\sup _{n} \mathrm{E}\left(\xi_{n} \mid \mathscr{G}\right) \leqslant \sup _{n} \mathrm{E}\left(\xi_{n} I_{\left\{\xi_{n} \leqslant c_{m}\right\}} \mid \mathscr{G}\right)+\varepsilon \leqslant c_{m}+\varepsilon<\infty .
\end{gathered}
$$

В силу того, что $\mathrm{E}\left(\xi_{n} \mid \mathscr{G}\right) \rightarrow \mathrm{E}(\xi \mid \mathscr{G})$, получаем $\mathrm{E}(\xi \mid \mathscr{G})<\infty$ (п.н.).

Необходимость. Положим $A=\{a: \mathrm{P}(\xi=a)>0\}$. Тогда

$$
\forall a \notin A \quad \xi_{n} I_{\left\{\xi_{n}<a\right\}} \rightarrow \xi I_{\{\xi<a\}} \quad \text { (п.н.). }
$$

Семейство случайных величин $\left\{\xi_{n} I_{\left\{\xi_{n}<a\right\}}\right\}_{n \geqslant 1}$ обладает свойством "равномерной интегригуемости”, и для него выполнена “достаточность” этой теоремы, и, следовательно,

$$
\left.\mathrm{E}\left(\xi_{n} I_{\left\{\xi_{n}<a\right\}} \mid \mathscr{G}\right) \rightarrow \mathrm{E}\left(\xi I_{\{\xi<a\}} \mid \mathscr{G}\right) \text { (п.н. }\right) .
$$


В силу того, что $\mathrm{E}\left(\xi_{n} \mid \mathscr{G}\right) \rightarrow \mathrm{E}(\xi \mid \mathscr{G})$ п.н.,

$$
\mathrm{E}\left(\xi_{n} I_{\left\{\xi_{n} \geqslant a\right\}} \mid \mathscr{G}\right) \rightarrow \mathrm{E}\left(\xi I_{\{\xi \geqslant a\}} \mid \mathscr{G}\right) \text { (п.н.). }
$$

Рассмотрим последовательность

$$
\left\{c_{m}\right\}_{m \geqslant 1}, \quad c_{m} \in \mathbb{R}^{+} \backslash A, \quad c_{m} \underset{m \rightarrow \infty}{\longrightarrow} \infty, \quad c_{m+1}>c_{m}, \quad m \geqslant 1 .
$$

Для каждой пары $c_{i}, c_{j}$ из $\left\{c_{m}\right\}_{m \geqslant 1}, c_{i}>c_{j}$, почти наверное (на $\left.G_{i, j}\right)$ выполнено:

$$
\mathrm{E}\left(\xi_{n} I_{\left\{\xi_{n} \geqslant c_{i}\right\}} \mid \mathscr{G}\right) \leqslant \mathrm{E}\left(\xi_{n} I_{\left\{\xi_{n} \geqslant c_{j}\right\}} \mid \mathscr{G}\right) .
$$

Пусть теперь $F \in \mathscr{F}, \mathrm{P}(F)=1,-$ такое множество, на котором:

$$
\begin{gathered}
\forall n \geqslant 1 \quad \mathrm{E}\left(\xi_{n} I_{\left\{\xi_{n} \geqslant a_{1}\right\}} \mid \mathscr{G}\right) \underset{a_{1} \rightarrow \infty}{\longrightarrow} 0, \\
\left.\forall m \geqslant 1 \mathrm{E}\left(\xi_{n} I_{\left\{\xi_{n} \geqslant c_{m}\right\}} \mid \mathscr{G}\right) \underset{n \rightarrow \infty}{\longrightarrow} \mathrm{E}\left(\xi I_{\{\xi \geqslant c}\right\} \mid \mathscr{G}\right), \\
\mathrm{E}\left(\xi I_{\left\{\xi \geqslant a_{0}\right\}} \mid \mathscr{G}\right) \underset{a_{0} \rightarrow \infty}{\longrightarrow} 0 .
\end{gathered}
$$

Пусть также

$$
\omega \in F \bigcap_{i, j=1}^{\infty} G_{i, j}
$$

Тогда

$$
\begin{gathered}
\forall \varepsilon>0 \quad \exists a_{0} \in\left\{c_{m}\right\}_{m \geqslant 1}: \quad \mathrm{E}\left(\xi I_{\left\{\xi \geqslant a_{0}\right\}} \mid \mathscr{G}\right)(\omega) \leqslant \frac{\varepsilon}{2}, \\
\exists M \quad \forall n>M: \quad \mathrm{E}\left(\xi_{n} I_{\left\{\xi_{n} \geqslant a_{0}\right\}} \mid \mathscr{G}\right)(\omega) \leqslant \mathrm{E}\left(\xi I_{\left\{\xi \geqslant a_{0}\right\}} \mid \mathscr{G}\right)(\omega)+\frac{\varepsilon}{2} \leqslant \varepsilon .
\end{gathered}
$$

Выберем $a_{1}>a_{0}, a_{1} \in\left\{c_{m}\right\}_{m \geqslant 1}$, таким, что при $n \leqslant M \mathrm{E}\left(\xi_{n} I_{\left\{\xi_{n} \geqslant a_{1}\right\}} \mid \mathscr{G}\right)(\omega) \leqslant \varepsilon$. Тогда

$$
\sup _{n} \mathrm{E}\left(\xi_{n} I_{\left\{\xi_{n} \geqslant a_{1}\right\}} \mid \mathscr{G}\right)(\omega) \leqslant \varepsilon .
$$

Тогда при $a>a_{1}, a \in\left\{c_{m}\right\}_{m \geqslant 1}$, получим:

$$
\sup _{n} \mathrm{E}\left(\xi_{n} I_{\left\{\xi_{n} \geqslant a\right\}} \mid \mathscr{G}\right)(\omega) \leqslant \varepsilon
$$

что и доказьвает теорему.

Автор хотел бы поблагодарить А. Н. Ширяева и А. А. Гущина за постановку задачи, внимание к работе и рекомендации по ее выполнению.

\section{СПИСОК ЛИТЕРАТУРЫ}

[1] Ширяев А. Н. Вероятность. М.: Наука, 1989. [2] Zheng Wei-an. // Z. Wahrscheinlichkeitstheorie verw. Geb. 1980. V. 53. P. 291-292. 